\title{
EQUAÇÕES PARA ESTIMAR O ESTOQUE DE CARBONO NO FUSTE DE ÁRVORES INDIVIDUAIS E EM PLANTIOS COMERCIAIS DE EUCALIPTO ${ }^{1}$
}

\author{
Carlos Pedro Boechat Soares ${ }^{2}$, Helio Garcia Leite ${ }^{2}$ e Eric Bastos Görgens ${ }^{3}$
}

\begin{abstract}
RESUMO - O objetivo deste trabalho foi ajustar equações para estimar o carbono presente no fuste de árvores individuais de eucalipto e o estoque de carbono, por unidade de área, em diferentes condições de plantio. Para isso, foram utilizadas 532 árvores para ajustar a equação referente ao modelo alométrico de Schumacher e Hall (1933) e 95 parcelas permanentes para ajustar as equações referentes aos modelos de crescimento e produção, incluindo as seguintes variáveis independentes: idade (I), área basal (B) e índice de local (S). Após as análises, verificou-se que as equações se ajustaram bem aos dados observados, fornecendo estimativas precisas.
\end{abstract}

Palavra-chave: Eucalipto, carbono, modelos de crescimento e produção e equação alométrica.

\section{EQUATIONS TO ESTIMATE CARBON STOCK IN STEMS OF INDIVIDUAL TREES AND STANDS OF EUCALYPTUS}

\begin{abstract}
The objective of this work was to adjust equations to estimate carbon in the stems of individual eucalyptus trees and carbon stock, by unit of area, in different planting conditions. For that, 532 trees were used to adjust the allometric model of Schumacher and Hall (1933) and 95 permanent plots to adjust equations related to growth and yield models, including the following independent variables: age (I), basal area $(B)$ and site index $(S)$. The analyses showed that the equations adjusted well to the observed data, providing accurate estimates.
\end{abstract}

Keywords: Eucalyptus, carbon stock, growth and yield models and allometric equation.

\section{INTRODUÇÃO}

A utilização de modelos para representar ou explicar uma realidade é uma estratégia muito utilizada em vários campos da ciência. Segundo Hasenauer (2000), modelos são apresentações simplificadas de uma realidade, em que o grau de detalhamento dos dados de entrada limita o detalhamento dos resultados.

Especificamente no setor florestal, os modelos de crescimento e produção volumétrica são utilizados no planejamento das atividades florestais. No entanto, o desenvolvimento e ajuste de modelos que quantifiquem o teor de carbono presente em uma floresta vêm se tornando uma necessidade, devido ao grande interesse com relação à captura de carbono por esses ecossistemas.

O interesse pela quantificação do estoque de carbono em florestas se deve à crescente demanda de alternativas que visem ao controle das emissões atmosféricas de $\mathrm{CO}_{2}$, entre elas: a diminuição da emissão desse gás para a atmosfera e a remoção do $\mathrm{CO}_{2}$ atmosférico por ecossistemas terrestres e pelos oceanos (SCHROEDER, 1992).

\footnotetext{
${ }^{1}$ Recebido em 28.04.2004 e aceito para publicação em 10.08.2005.

${ }^{2}$ Departamento de Engenharia Florestal da Universidade Federal de Viçosa (UFV). E.mail:<csoares@ufv.br $>$; $<$ hgleite@ufv.br>.

${ }^{3}$ Programa de Pós-Graduação em Ciência Florestal da UFV.
} 
Nesse contexto, as florestas têm papel importante, pois são capazes de armazenar grandes quantidades de carbono em seus diferentes compartimentos. Segundo Grainger, citado por Schroeder (1992), somente os trópicos possuem cerca de 2 (dois) bilhões de hectares de áreas degradadas ou não florestadas, das quais 758 milhões poderiam ser reflorestadas.

A preocupação em relação à elevação da concentração de $\mathrm{CO} 2$ na atmosfera é legítima, uma vez que a conseqüência desse fato é o aquecimento atmosférico global, denominado efeito-estufa, o qual pode causar, segundo os especialistas, mudanças permanentes no clima, alterando o regime dos ventos, a pluviosidade e a circulação dos oceanos (SOARES e OLIVEIRA, 2002).

Embora as florestas possam desempenhar importante papel na captura do $\mathrm{CO} 2$ atmosférico, a atividade florestal também é responsável pelo aumento da concentração desse gás. De acordo com o uso da floresta, todo o carbono acumulado é devolvido à atmosfera, por exemplo na combustão da madeira para sua transformação em carvão ou no processo de polpação para a produção de papel.

Nesse sentido, existem muitas incertezas sobre a real contribuição para o fluxo de $\mathrm{CO} 2$ dos processos de desflorestamento e reflorestamento, uma vez que estimativas sobre a quantidade de carbono presente nas florestas ainda são feitas, utilizando-se métodos pouco precisos (COOPER, 1983; BROWN e LUGO, 1984; BROWN et al., 1986). Assim, este trabalho foi desenvolvido com o objetivo de ajustar equações para estimar o carbono presente no fuste de árvores individuais de eucalipto e o estoque de carbono, por unidade de área, em diferentes condições de plantio.

\section{MATERIAL E MÉTODOS}

Os dados para a realização deste estudo são provenientes de plantios de Eucalyptus grandis W. Hill ex Maiden, com idades entre 32 e 80 meses, pertencentes à Cenibra S/A (Celulose Nipo-Brasileira S/A), localizados na região leste do Estado de Minas Gerais.

Para o ajuste das equações de carbono para árvores individuais foram selecionadas aleatoriamente 532 árvores, distribuídas em diferentes classes de tamanho (Quadro 1). Em cada árvore-amostra, mediram-se o DAP (diâmetro com casca a 1,30 m do solo) e a altura total da árvore, bem como se realizou o procedimento de cubagem rigorosa para obtenção do volume do fuste com e sem casca, pela aplicação sucessiva da expressão de Smalian, em seções de $1 \mathrm{~m}$ de comprimento (HUSCH et al., 1993). Além disso, foram retirados discos de madeira e casca para determinação das densidades básicas da madeira e da casca (VITAL, 1984), que, multiplicadas pelos respectivos volumes, forneceram as estimativas de biomassa da madeira e casca do fuste.

Quadro 1 - Número de árvores-amostra por classe de diâmetro e altura Table 1 -Number of sample trees per diameter and height classes

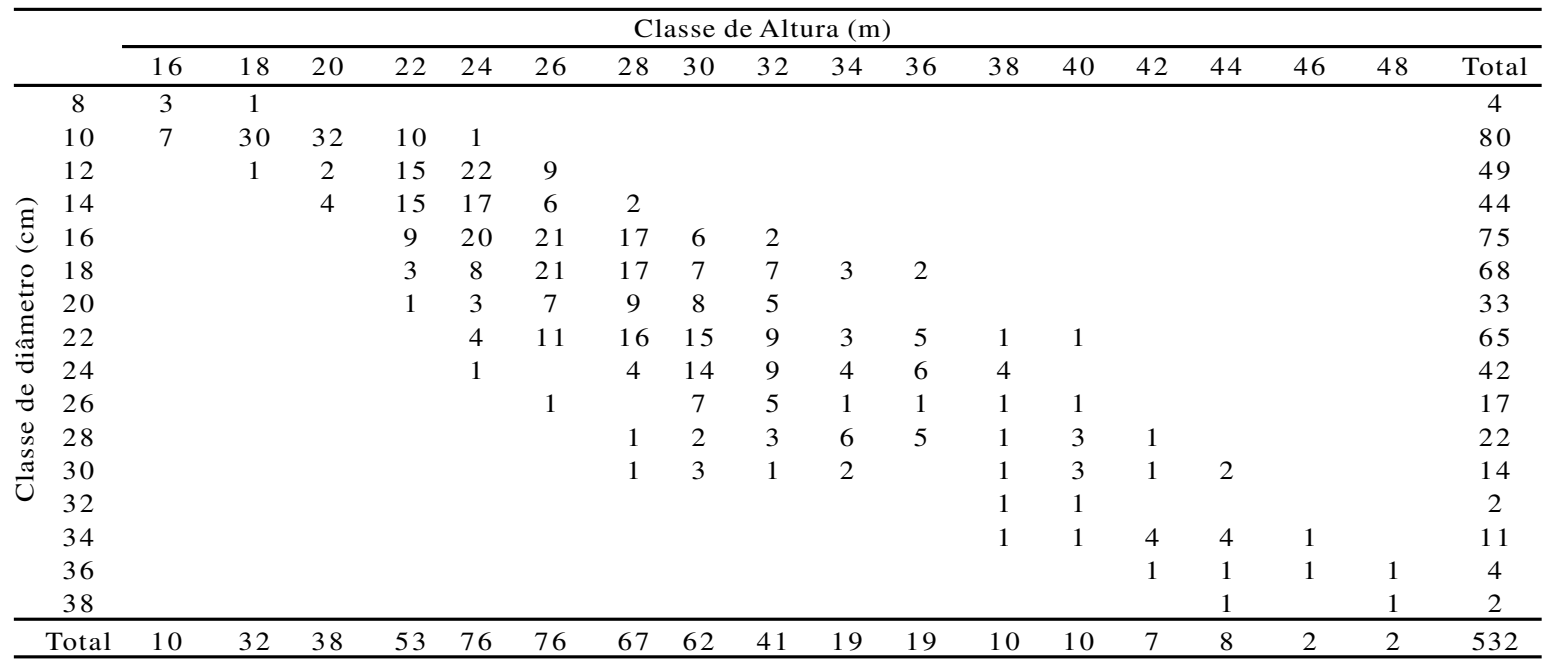

R. Árvore, Viçosa-MG, v.29, n.5, p.711-718, 2005 
Para obter as estimativas do carbono estocado no fuste de cada árvore-amostra (madeira e casca), multiplicou-se a estimativa da biomassa por 0,5 , ou seja, considerou-se que $50 \%$ da biomassa é composta por carbono (HIGUCHI e CARVALHO JR., 1994).

As estimativas do carbono estocado nos fustes das árvores-amostra e as respectivas medidas de DAP e altura total (Ht) foram utilizadas no ajuste de equações referentes ao modelo linearizado de Schumacher e Hall (1933), cuja relação funcional é assim definida:

$$
\mathrm{LnY}=\beta_{0}+\beta_{1} \cdot \operatorname{LnDAP}+\beta_{2} \cdot \mathrm{LnHt}+\varepsilon
$$

em que:

$$
\begin{aligned}
& \mathrm{Ln}=\text { logaritmo neperiano; } \\
& \begin{aligned}
\mathrm{Y}= & \text { carbono total no fuste (madeira e casca) }- \\
& \mathrm{CF}, \text { ou carbono na madeira }-\mathrm{CM}, \text { em } \mathrm{kg} ;
\end{aligned} \\
& \beta_{\mathrm{i}}=\text { parâmetros do modelo, para } \mathrm{i}=1,2 \text { e } 3 ; \mathrm{e} \\
& \varepsilon=\text { erro aleatório. }
\end{aligned}
$$

Para avaliar a precisão das equações foram utilizados o coeficiente de determinação $\left(\mathrm{R}^{2}\right)$, o erro-padrão da estimativa $\left(\mathrm{S}_{\mathrm{y} . \mathrm{x}}\right)$ e a análise gráfica de resíduos porcentuais (R\%), obtidos por:

$$
\mathrm{R} \%=\frac{Y_{e s t}-Y_{o b s}}{Y_{o b s}} \cdot 100
$$

Utilizando dados de 95 parcelas permanentes, medidas entre 1996 e 2000, e as equações de carbono para árvores individuais previamente ajustadas, procedeu-se ao ajuste das equações referentes aos seguintes modelos de crescimento e produção (CAMPOS e LEITE, 2002):

$$
\begin{aligned}
& \mathrm{LnY}=\beta_{0}+\beta_{1} / \mathrm{I}+\varepsilon \\
& \mathrm{LnY}=\beta_{0}+\beta_{1} /(\mathrm{I} \cdot \mathrm{S})+\varepsilon \\
& \mathrm{LnY}_{2}=\beta_{0}+\beta_{1} \cdot 1 / \mathrm{I}_{2}+\beta_{2} \cdot \mathrm{S}+\beta_{3} \cdot \mathrm{LnB}_{2}+\varepsilon \\
& \mathrm{LnB}_{2}=\mathrm{Ln} \mathrm{B}_{1} \cdot\left(\mathrm{I}_{1} / \mathrm{I}_{2}\right)+\beta_{4} \cdot\left(1-\mathrm{I}_{1} / \mathrm{I}_{2}\right)+\beta_{5} \cdot\left(1-\mathrm{I}_{1} / \mathrm{I}_{2}\right) \cdot \mathrm{S}+\varepsilon(5 \mathrm{~b}) \\
& \mathrm{em} \text { que: } \\
& \mathrm{Ln}=\text { logaritmo neperiano; } \\
& \mathrm{I}=\text { idade, em meses; }
\end{aligned}
$$

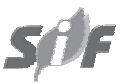

$$
\begin{aligned}
& I_{1} \text { e } I_{2}=\text { idade presente e idade futura, } \\
& \text { respectivamente, em meses; } \\
& \mathrm{B}_{1} \text { e } \mathrm{B}_{2}=\text { área basal presente e área basal futura, } \\
& \text { respectivamente, em } \mathrm{m}^{2} / \mathrm{ha} \text {; }
\end{aligned}
$$

A avaliação da precisão das equações referentes aos modelos descritos anteriormente foi realizada, utilizando-se também o coeficiente de determinação $\left(\mathrm{R}^{2}\right)$, o erro-padrão da estimativa $\left(\mathrm{S}_{\mathrm{y} . \mathrm{x}}\right)$ e a análise gráfica de resíduos porcentuais, além da determinação das idades técnicas de colheita (ITC) nas três classes de produtividade.

\section{RESULTADOS E DISCUSSÃO}

Analisando os coeficientes de determinação $\left(R^{2}\right)$ das equações ajustadas para estimar o carbono total no fuste (CF) e o carbono na madeira (CM) de árvores individuais de eucalipto, verifica-se que as equações se ajustaram bem aos dados observados. Além disso, todos os coeficientes foram significativos em nível de $95 \%$ de probabilidade (*), pelo teste “t”, de Student.

\section{Carbono Total no Fuste (CF)}

$$
\begin{aligned}
& \mathrm{LnCF}=-4,833265^{*}+1,8284191^{*} . \mathrm{LnDAP}+1,1724611^{*} \cdot \mathrm{LnHt} \\
& \mathrm{R}^{2}=98,02 \% ; \quad \mathrm{S}_{\mathrm{y} \cdot \mathrm{x}}= \pm 0,1244 \ln (\mathrm{kg})
\end{aligned}
$$

\section{Carbono na Madeira (CM)}

$$
\mathrm{LnCM}=-5,107646^{*}+1,7947037^{*} . \mathrm{LnDAP}+1,2604764^{*} \text {.LnHt }
$$$$
\mathrm{R}^{2}=97,96 \% ; \quad \mathrm{S}_{\mathrm{y} . \mathrm{x}}= \pm 0,1273 \ln (\mathrm{kg})
$$

Observando a distribuição dos resíduos das equações na Figura 1, verifica-se a exatidão das estimativas, pela amplitude dos valores dos resíduos. Quanto à homogeneidade da distribuição dos resíduos porcentuais, notou-se tendência de as equações superestimarem o carbono das árvores com DAP maiores que $30 \mathrm{~cm}$.

R. Árvore, Viçosa-MG, v.29, n.5, p.711-718, 2005 


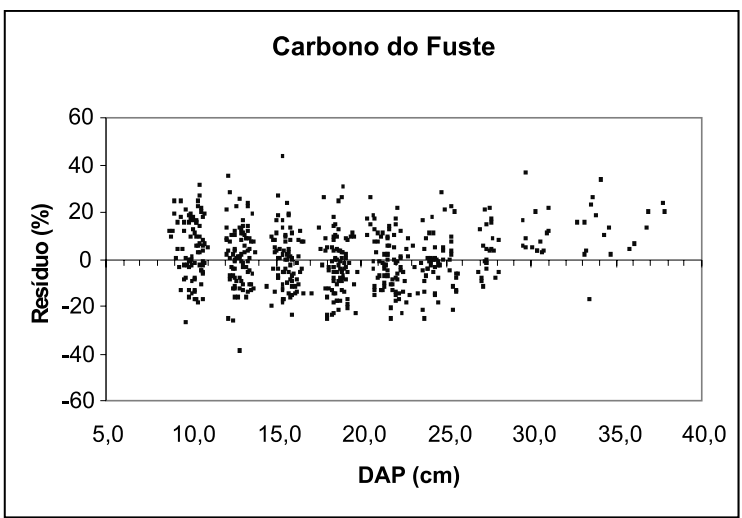

(A)

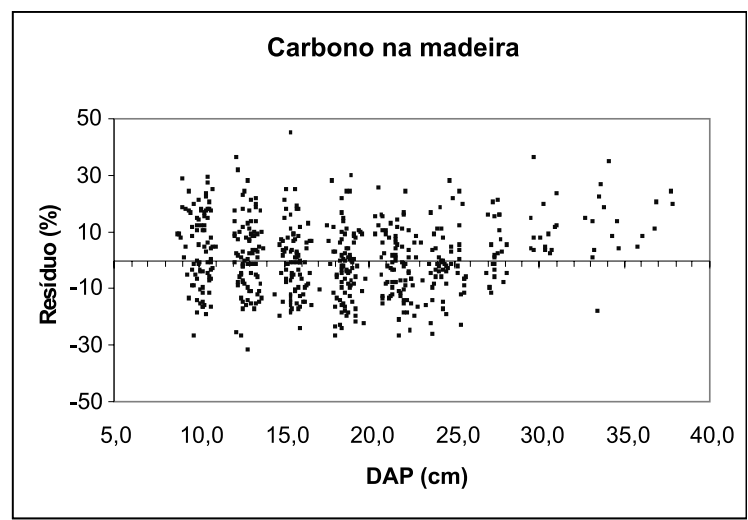

(B)

Figura 1 - Distribuição dos resíduos porcentuais nas equações que estimam o carbono total no fuste (A) e o carbono na madeira (B) de árvores individuais.

Figure 1 -Distribution of the percentile residuals in the equations that estimate total carbon outside bark (A) and inside bark (B) for individual trees.

Considerando somente os modelos de crescimento e produção, que incluem as variáveis independentes idade (I) e índice de local (S), obtiveram-se as seguintes equações para o estoque de carbono total no fuste (CF) e para o estoque de carbono na madeira (CM):

$$
\begin{array}{cl}
\mathrm{LnCF}=12,2032^{*}-87,2985^{*} / \mathrm{I} ; & \mathrm{R}^{2}=51,79 \% ; \\
& \mathrm{S}_{\mathrm{y} \cdot \mathrm{x}}= \pm 0,2605 \ln (\mathrm{kg} / \mathrm{ha}) \\
\mathrm{LnCM}=12,1443^{*}-89,2677^{*} / \mathrm{I} ; & \mathrm{R}^{2}=51,84 \% \\
& \mathrm{~S}_{\mathrm{y} \cdot \mathrm{x}}= \pm 0,2662 \ln (\mathrm{kg} / \mathrm{ha}) \\
\mathrm{LnCF}=12,2026^{*}-2194,4^{*} /(\mathrm{I} . \mathrm{S}) ; & \mathrm{R}^{2}=79,44 \% \\
\mathrm{~S}_{\mathrm{y} \cdot \mathrm{x}}= \pm 0,1702 \ln (\mathrm{kg} / \mathrm{ha}) \\
\mathrm{LnCM}=12,1446^{*}-2245,36^{*} /(\mathrm{I} . \mathrm{S}) ; \quad \mathrm{R}^{2}=79,61 \% \\
\mathrm{~S}_{\mathrm{y} \cdot \mathrm{x}}= \pm 0,1732 \ln (\mathrm{kg} / \mathrm{ha})
\end{array}
$$

As variáveis independentes das equações ajustadas foram todas significativas a $95 \%$ de probabilidade (*), tanto para a estimação do estoque de carbono total no fuste quanto para o estoque de carbono na madeira, pelo teste "t", de Student. A inclusão da variável índice de local (S) aumentou significativamente a precisão das equações, quando comparadas com aquelas que possuíam apenas a idade (I) como variável independente, haja vista o aumento do coeficiente de determinação e a diminuição do erro-padrão da estimativa.

Os gráficos de resíduos, na Figura 2, indicam não haver tendência na estimação do estoque de carbono por hectare. No entanto, percebe-se maior exatidão das estimativas para as equações que apresentavam as variáveis independentes I e $\mathrm{S}$, pela menor amplitude dos resíduos porcentuais.

Para o modelo de crescimento e produção de Clutter (1963) - modelos 5a e 5b, obtiveram-se as seguintes equações:

$\mathrm{LnB}_{2}=\mathrm{LnB}_{1} \cdot\left(\mathrm{I}_{1} / \mathrm{I}_{2}\right)+3,405011^{*} .\left(1-\mathrm{I}_{1} / \mathrm{I}_{2}\right)+0,010680^{\mathrm{ns}} .\left(1-\mathrm{I}_{1} / \mathrm{I}_{2}\right) \cdot \mathrm{S}$

$$
\mathrm{R}^{2}=96,56 \% \quad \mathrm{~S}_{\mathrm{y} . \mathrm{x}}= \pm 0,0444 \ln \left(\mathrm{m}^{2} / \mathrm{ha}\right)
$$

$\mathrm{LnCF}_{2}=6,983563^{*}-20,802700^{*} .1 / \mathrm{I}_{2}+0,030827^{*} . \mathrm{S}+$ $1,130176 * \mathrm{LnB}_{2} \quad \mathrm{R}^{2}=98,61 \% \quad \mathrm{~S}_{\mathrm{y} . \mathrm{x}}= \pm 0,0445 \ln (\mathrm{kg} / \mathrm{ha})$ $\mathrm{LnCM}_{2}=6,844098^{*}-22,052370^{*} .1 / \mathrm{I}_{2}+0,032648^{*} . \mathrm{S}+$ $1,137806^{*} \cdot \mathrm{LnB}_{2} \quad \mathrm{R}^{2}=98,50 \% \quad \mathrm{~S}_{\mathrm{y} . \mathrm{x}}= \pm 0,0472 \ln (\mathrm{kg} / \mathrm{ha})$

Pela análise dos coeficientes de determinação das equações que projetam a área basal por hectare $\left(\mathrm{B}_{2}\right)$, o estoque futuro de carbono no fuste por hectare $\left(\mathrm{CF}_{2}\right)$ e o estoque futuro de carbono na madeira por hectare $\left(\mathrm{CM}_{2}\right)$, verificou-se que as equações também se ajustaram bem a dados observados. Todas as variáveis, com exceção da variável " $\left(1-I_{1} / I_{2}\right)$.S" na equação de área basal, foram significativas a $95 \%$ de probabilidade $(*)$, pelo teste "t" de Student.

Os gráficos de resíduos (Figura 3), por sua vez, indicam não haver tendência na estimação dos estoques de carbono dos plantios de eucalipto, verificando-se, nessa figura, exatidão entre os valores observados e estimados, pela amplitude dos resíduos porcentuais. 

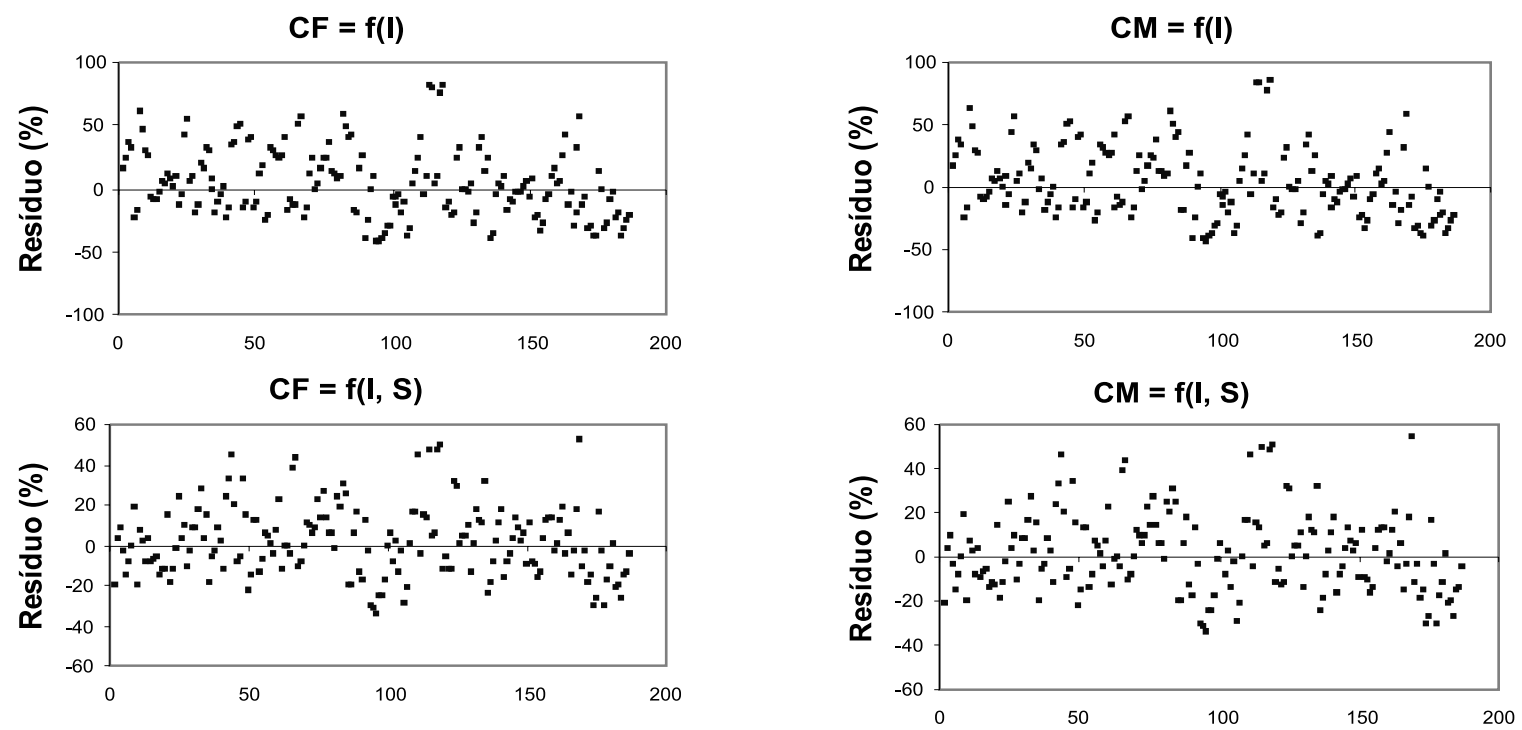

Figura 2 - Gráficos dos resíduos porcentuais referentes a equações que incluem as variáveis independentes idade (I) e idade e índice de local (I,S), do carbono total no fuste (CF) e na madeira (CM).

Figure 2 -Graphs of the percentile residuals for equations that include the independent variables age (I); age and site index $(I, S)$, for the total carbon stock outside bark (CF) and inside bark (CM).

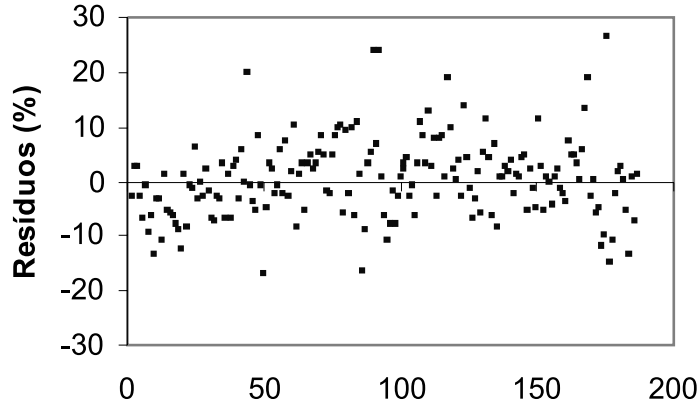

(A)

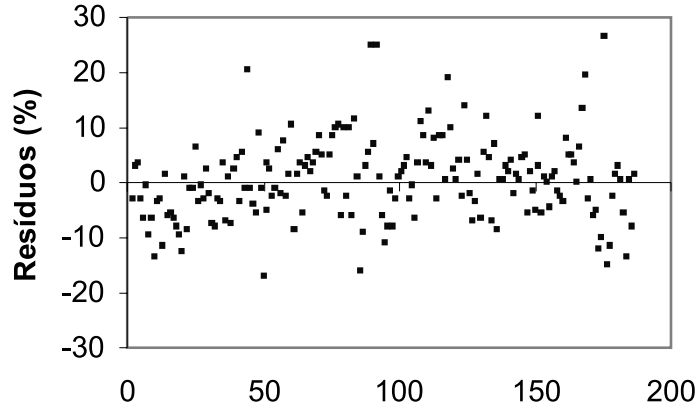

(B)

Figura 3 - Gráfico dos resíduos porcentuais das equações referentes ao modelo de Clutter (1963), para o carbono total no fuste (A) e na madeira (B).

Figure 3 - Graphs of the percentile residuals for equations of the CLUTTER model (1963), for the total carbon stock outside bark (A) and inside bark (B).

De posse das equações referentes ao modelo de Clutter (1963), elaboraram-se as tabelas de produção de densidade variável (Quadros 2 e 3), considerando três classes de produtividade: $\mathrm{S}=21,5 ; \mathrm{S}=26,5$; e $\mathrm{S}=31,5$.

Com as estimativas dos Quadros 2 e 3, elaboraramse os gráficos apresentados na Figura 4, os quais mostram as idades técnicas de colheita (ITC's) nas três classes de produtividade. De acordo com a Figura 4 , verifica-se menor ITC nos melhores locais (maior índice de local -S), tanto no estoque total de carbono no fuste quanto no estoque de carbono na madeira. Cabe ressaltar que as idades técnicas de colheita foram ligeiramente menores quando se considera o estoque total de carbono no fuste (madeira + casca).

R. Árvore, Viçosa-MG, v.29, n.5, p.711-718, 2005 
Quadro 2 - Tabela de produção do povoamentos de Eucalyptus grandis, em que: $\mathrm{CF}=$ estoque total de carbono no fuste (madeira+casca) em kg/ha; IMM = incremento médio mensal, em kg/ha.mês; e B = área basal por hectare

Table 2 - Yield for stands of Eucalyptus grandis, where: CF = total carbon stock outside bark, in $\mathrm{kg} / \mathrm{ha} ; \mathrm{IMM}=\mathrm{mean}$ monthly increment, (kg/ha.month); B = basal area per hectare

\begin{tabular}{|c|c|c|c|c|c|c|c|c|c|}
\hline \multirow[t]{2}{*}{ Idade (I) } & \multicolumn{3}{|c|}{$\mathrm{S}=21,5$} & \multicolumn{3}{|c|}{$\mathrm{S}=26,5$} & \multicolumn{3}{|c|}{$\mathrm{S}=31,5$} \\
\hline & $\mathrm{B}$ & $\mathrm{CF}$ & IMM & $\mathrm{B}$ & $\mathrm{CF}$ & IMM & B & $\mathrm{CF}$ & IMM \\
\hline 32 & 7,87 & 11240,94 & 351,28 & 10,29 & 17773,33 & 555,42 & 12,99 & 26978,09 & 843,07 \\
\hline 33 & 8,25 & 12098,74 & 366,63 & 10,73 & 18988,91 & 575,42 & 13,47 & 28646,59 & 868,08 \\
\hline 34 & 8,63 & 12965,80 & 381,35 & 11,15 & 20208,83 & 594,38 & 13,92 & 30311,09 & 891,50 \\
\hline 35 & 9,00 & 13840,14 & 395,43 & 11,56 & 21430,73 & 612,31 & 14,37 & 31968,97 & 913,40 \\
\hline 36 & 9,37 & 14719,99 & 408,89 & 11,97 & 22652,52 & 629,24 & 14,81 & 33617,92 & 933,83 \\
\hline 37 & 9,73 & 15603,70 & 421,72 & 12,36 & 23872,31 & 645,20 & 15,23 & 35255,95 & 952,86 \\
\hline 38 & 10,08 & 16489,78 & 433,94 & 12,75 & 25088,43 & 660,22 & 15,65 & 36881,32 & 970,56 \\
\hline 39 & 10,43 & 17376,89 & 445,56 & 13,13 & 26299,40 & 674,34 & 16,05 & 38492,56 & 986,99 \\
\hline 40 & 10,77 & 18263,82 & 456,60 & 13,50 & 27503,93 & 687,60 & 16,44 & 40088,39 & 1002,21 \\
\hline 41 & 11,11 & 19149,45 & 467,06 & 13,86 & 28700,85 & 700,02 & 16,82 & 41667,73 & 1016,29 \\
\hline 42 & 11,44 & 20032,81 & 476,97 & 14,22 & 29889,17 & 711,65 & 17,20 & 43229,67 & 1029,28 \\
\hline 43 & 11,76 & 20913,00 & 486,35 & 14,56 & 31068,02 & 722,51 & 17,56 & 44773,46 & 1041,24 \\
\hline 44 & 12,08 & 21789,24 & 495,21 & 14,90 & 32236,62 & 732,65 & 17,91 & 46298,48 & 1052,24 \\
\hline 45 & 12,39 & 22660,82 & 503,57 & 15,23 & 33394,34 & 742,10 & 18,26 & 47804,23 & 1062,32 \\
\hline 46 & 12,69 & 23527,10 & 511,46 & 15,56 & 34540,60 & 750,88 & 18,59 & 49290,32 & 1071,53 \\
\hline 47 & 12,99 & 24387,54 & 518,88 & 15,87 & 35674,94 & 759,04 & 18,92 & 50756,44 & 1079,92 \\
\hline 48 & 13,28 & 25241,64 & 525,87 & 16,18 & 36796,96 & 766,60 & 19,24 & 52202,37 & 1087,55 \\
\hline 49 & 13,57 & 26088,97 & 532,43 & 16,48 & 37906,32 & 773,60 & 19,55 & 53627,98 & 1094,45 \\
\hline 50 & 13,85 & 26929,16 & 538,58 & 16,77 & 39002,76 & 780,06 & 19,85 & 55033,17 & 1100,66 \\
\hline 51 & 14,13 & 27761,87 & 544,35 & 17,06 & 40086,07 & 786,00 & 20,14 & 56417,93 & 1106,23 \\
\hline 52 & 14,40 & 28586,83 & 549,75 & 17,34 & 41156,07 & 791,46 & 20,43 & 57782,28 & 1111,20 \\
\hline 53 & 14,66 & 29403,80 & 554,79 & 17,62 & 42212,66 & 796,47 & 20,71 & 59126,29 & 1115,59 \\
\hline 54 & 14,93 & 30212,57 & 559,49 & 17,89 & 43255,74 & 801,03 & 20,99 & 60450,05 & 1119,45 \\
\hline 55 & 15,18 & 31012,96 & 563,87 & 18,15 & 44285,26 & 805,19 & 21,26 & 61753,71 & 1122,79 \\
\hline 56 & 15,43 & 31804,85 & 567,94 & 18,41 & 45301,22 & 808,95 & 21,52 & 63037,43 & 1125,67 \\
\hline 57 & 15,68 & 32588,11 & 571,72 & 18,66 & 46303,62 & 812,34 & 21,77 & 64301,40 & 1128,09 \\
\hline 58 & 15,92 & 33362,66 & 575,22 & 18,91 & 47292,50 & 815,39 & 22,02 & 65545,83 & 1130,10 \\
\hline 59 & 16,15 & 34128,44 & 578,45 & 19,15 & 48267,91 & 818,10 & 22,27 & 66770,94 & 1131,71 \\
\hline 60 & 16,38 & 34885,39 & 581,42 & 19,39 & 49229,92 & 820,50 & 22,50 & 67976,98 & 1132,95 \\
\hline 61 & 16,61 & 35633,50 & 584,16 & 19,62 & 50178,63 & 822,60 & 22,74 & 69164,19 & 1133,84 \\
\hline 62 & 16,83 & 36372,74 & 586,66 & 19,84 & 51114,14 & 824,42 & 22,96 & 70332,84 & 1134,40 \\
\hline 63 & 17,05 & 37103,13 & 588,94 & 20,07 & 52036,56 & 825,98 & $\underline{23,19}$ & $\underline{71483,21}$ & $\underline{1134,65}$ \\
\hline 64 & 17,26 & 37824,67 & 591,01 & 20,28 & 52946,04 & 827,28 & 23,40 & 72615,57 & 1134,62 \\
\hline 65 & 17,47 & 38537,40 & 592,88 & 20,50 & 53842,69 & 828,35 & 23,62 & 73730,20 & 1134,31 \\
\hline 66 & 17,68 & 39241,36 & 594,57 & 20,70 & 54726,67 & 829,19 & 23,83 & 74827,39 & 1133,75 \\
\hline 67 & 17,88 & 39936,59 & 596,07 & 20,91 & 55598,14 & 829,82 & 24,03 & 75907,43 & 1132,95 \\
\hline 68 & 18,08 & 40623,14 & 597,40 & 21,11 & 56457,24 & 830,25 & 24,23 & 76970,62 & 1131,92 \\
\hline 69 & 18,28 & 41301,09 & 598,57 & 21,30 & 57304,14 & 830,49 & 24,42 & 78017,23 & 1130,68 \\
\hline 70 & 18,47 & 41970,51 & 599,58 & $\underline{21,50}$ & $\underline{\mathbf{5 8 1 3 9 , 0 0}}$ & $\underline{830,56}$ & 24,62 & 79047,56 & 1129,25 \\
\hline 71 & 18,65 & 42631,46 & 600,44 & 21,69 & 58962,00 & 830,45 & 24,80 & 80061,91 & 1127,63 \\
\hline 72 & 18,84 & 43284,03 & 601,17 & 21,87 & 59773,31 & 830,18 & 24,99 & 81060,56 & 1125,84 \\
\hline 73 & 19,02 & 43928,30 & 601,76 & 22,05 & 60573,10 & 829,77 & 25,17 & 82043,80 & 1123,89 \\
\hline 74 & 19,20 & 44564,36 & 602,22 & 22,23 & 61361,54 & 829,21 & 25,34 & 83011,90 & 1121,78 \\
\hline 75 & 19,37 & 45192,30 & 602,56 & 22,40 & 62138,81 & 828,52 & 25,51 & 83965,16 & 1119,54 \\
\hline 76 & 19,54 & 45812,22 & 602,79 & 22,58 & 62905,09 & 827,70 & 25,68 & 84903,86 & 1117,16 \\
\hline 77 & 19,71 & 46424,21 & 602,91 & 22,74 & 63660,54 & 826,76 & 25,85 & 85828,26 & 1114,65 \\
\hline 78 & $\underline{\mathbf{1 9 , 8 8}}$ & $\underline{47028,38}$ & $\underline{602,93}$ & 22,91 & 64405,35 & 825,71 & 26,01 & 86738,63 & 1112,03 \\
\hline 79 & $\overline{20,04}$ & $\overline{47624,81}$ & $\overline{602,85}$ & 23,07 & 65139,70 & 824,55 & 26,17 & 87635,25 & 1109,31 \\
\hline 80 & 20,20 & 48213,61 & 602,67 & 23,23 & 65863,74 & 823,30 & 26,33 & 88518,38 & 1106,48 \\
\hline
\end{tabular}

R. Árvore, Viçosa-MG, v.29, n.5, p.711-718, 2005 
Equações para estimar o estoque de carbono no ...

Quadro 3 - Tabela de produção do povoamentos de Eucalyptus grandis, em que: CM= estoque total de carbono na madeira, em $\mathrm{kg} / \mathrm{ha} ; \mathrm{IMM}=$ incremento médio mensal, em kg/ha.mês; e B = área basal por hectare

Table 3 - Yield for stands of Eucalyptus grandis, where: CM = total carbon stock inside bark, in $\mathrm{kg} / \mathrm{ha}$; IMM = mean monthly increment, (kg/ha.month); $B$ = basal area per hectare

\begin{tabular}{|c|c|c|c|c|c|c|c|c|c|}
\hline \multirow[t]{2}{*}{ Idade (I) } & \multicolumn{3}{|c|}{$\mathrm{S}=21,5$} & \multicolumn{3}{|c|}{$\mathrm{S}=26,5$} & \multicolumn{3}{|c|}{$\mathrm{S}=31,5$} \\
\hline & B & $\mathrm{CM}$ & IMM & B & $\mathrm{CM}$ & IMM & B & $\mathrm{CM}$ & IMM \\
\hline 32 & 7,87 & 9933,71 & 310,43 & 10,29 & 15882,66 & 496,33 & 12,99 & 24372,02 & 761,63 \\
\hline 33 & 8,25 & 10708,31 & 324,49 & 10,73 & 16994,35 & 514,98 & 13,47 & 25917,04 & 785,36 \\
\hline 34 & 8,63 & 11492,44 & 338,01 & 11,15 & 18111,64 & 532,70 & 13,92 & 27460,54 & 807,66 \\
\hline 35 & 9,00 & 12284,28 & 350,98 & 11,56 & 19232,27 & 549,49 & 14,37 & 28999,94 & 828,57 \\
\hline 36 & 9,37 & 13082,17 & 363,39 & 11,97 & 20354,24 & 565,40 & 14,81 & 30532,97 & 848,14 \\
\hline 37 & 9,73 & 13884,56 & 375,26 & 12,36 & 21475,74 & 580,43 & 15,23 & 32057,66 & 866,42 \\
\hline 38 & 10,08 & 14690,08 & 386,58 & 12,75 & 22595,17 & 594,61 & 15,65 & 33572,27 & 883,48 \\
\hline 39 & 10,43 & 15497,45 & 397,37 & 13,13 & 23711,08 & 607,98 & 16,05 & 35075,30 & 899,37 \\
\hline 40 & 10,77 & 16305,51 & 407,64 & 13,50 & 24822,19 & 620,55 & 16,44 & 36565,46 & 914,14 \\
\hline 41 & 11,11 & 17113,22 & 417,40 & 13,86 & 25927,40 & 632,38 & 16,82 & 38041,65 & 927,85 \\
\hline 42 & 11,44 & 17919,64 & 426,66 & 14,22 & 27025,69 & 643,47 & 17,20 & 39502,91 & 940,55 \\
\hline 43 & 11,76 & 18723,91 & 435,44 & 14,56 & 28116,19 & 653,86 & 17,56 & 40948,45 & 952,29 \\
\hline 44 & 12,08 & 19525,28 & 443,76 & 14,90 & 29198,15 & 663,59 & 17,91 & 42377,60 & 963,13 \\
\hline 45 & 12,39 & 20323,05 & 451,62 & 15,23 & 30270,91 & 672,69 & 18,26 & 43789,81 & 973,11 \\
\hline 46 & 12,69 & 21116,63 & 459,06 & 15,56 & 31333,88 & 681,17 & 18,59 & 45184,64 & 982,27 \\
\hline 47 & 12,99 & 21905,45 & 466,07 & 15,87 & 32386,58 & 689,08 & 18,92 & 46561,73 & 990,68 \\
\hline 48 & 13,28 & 22689,05 & 472,69 & 16,18 & 33428,59 & 696,43 & 19,24 & 47920,80 & 998,35 \\
\hline 49 & 13,57 & 23466,99 & 478,92 & 16,48 & 34459,56 & 703,26 & 19,55 & 49261,66 & 1005,34 \\
\hline 50 & 13,85 & 24238,89 & 484,78 & 16,77 & 35479,19 & 709,58 & 19,85 & 50584,18 & 1011,68 \\
\hline 51 & 14,13 & 25004,42 & 490,28 & 17,06 & 36487,24 & 715,44 & 20,14 & 51888,26 & 1017,42 \\
\hline 52 & 14,40 & 25763,31 & 495,45 & 17,34 & 37483,52 & 720,84 & 20,43 & 53173,88 & 1022,57 \\
\hline 53 & 14,66 & 26515,30 & 500,29 & 17,62 & 38467,88 & 725,81 & 20,71 & 54441,04 & 1027,19 \\
\hline 54 & 14,93 & 27260,17 & 504,82 & 17,89 & 39440,20 & 730,37 & 20,99 & 55689,81 & 1031,29 \\
\hline 55 & 15,18 & 27997,74 & 509,05 & 18,15 & 40400,42 & 734,55 & 21,26 & 56920,26 & 1034,91 \\
\hline 56 & 15,43 & 28727,87 & 513,00 & 18,41 & 41348,47 & 738,37 & 21,52 & 58132,51 & 1038,08 \\
\hline 57 & 15,68 & 29450,42 & 516,67 & 18,66 & 42284,34 & 741,83 & 21,77 & 59326,69 & 1040,82 \\
\hline 58 & 15,92 & 30165,30 & 520,09 & 18,91 & 43208,04 & 744,97 & 22,02 & 60502,97 & 1043,15 \\
\hline 59 & 16,15 & 30872,42 & 523,26 & 19,15 & 44119,58 & 747,79 & 22,27 & 61661,51 & 1045,11 \\
\hline 60 & 16,38 & 31571,72 & 526,20 & 19,39 & 45019,02 & 750,32 & 22,50 & 62802,52 & 1046,71 \\
\hline 60 & 16,38 & 31571,72 & 526,20 & 19,39 & 45019,02 & 750,32 & 22,50 & 62802,52 & 1046,71 \\
\hline 61 & 16,61 & 32263,16 & 528,90 & 19,62 & 45906,40 & 752,56 & 22,74 & 63926,20 & 1047,97 \\
\hline 62 & 16,83 & 32946,71 & 531,40 & 19,84 & 46781,81 & 754,55 & 22,96 & 65032,78 & 1048,92 \\
\hline 63 & 17,05 & 33622,36 & 533,69 & 20,07 & 47645,33 & 756,28 & 23,19 & 66122,47 & 1049,56 \\
\hline 64 & 17,26 & 34290,10 & 535,78 & 20,28 & 48497,07 & 757,77 & 23,40 & 67195,52 & 1049,93 \\
\hline 65 & 17,47 & 34949,95 & 537,69 & 20,50 & 49337,12 & 759,03 & 23,62 & 68252,17 & 1050,03 \\
\hline 66 & 17,68 & 35601,93 & 539,42 & 20,70 & 50165,62 & 760,09 & $\underline{23,83}$ & 69292,67 & $\underline{1049,89}$ \\
\hline 67 & 17,88 & 36246,07 & 540,99 & 20,91 & 50982,67 & 760,94 & 24,03 & 70317,26 & 1049,51 \\
\hline 68 & 18,08 & 36882,40 & 542,39 & 21,11 & 51788,42 & 761,59 & 24,23 & 71326,20 & 1048,91 \\
\hline 69 & 18,28 & 37510,98 & 543,64 & 21,30 & 52583,00 & 762,07 & 24,42 & 72319,75 & 1048,11 \\
\hline 70 & 18,47 & 38131,86 & 544,74 & 21,50 & 53366,54 & 762,38 & 24,62 & 73298,16 & 1047,12 \\
\hline 71 & 18,65 & 38745,09 & 545,71 & 21,69 & 54139,21 & 762,52 & 24,80 & 74261,70 & 1045,94 \\
\hline 72 & 18,84 & 39350,74 & 546,54 & $\underline{21,87}$ & $\underline{54901,13}$ & $\underline{762,52}$ & 24,99 & 75210,61 & 1044,59 \\
\hline 73 & 19,02 & 39948,88 & 547,24 & 22,05 & 55652,46 & 762,36 & 25,17 & 76145,15 & 1043,08 \\
\hline 74 & 19,20 & 40539,58 & 547,83 & 22,23 & 56393,35 & 762,07 & 25,34 & 77065,58 & 1041,43 \\
\hline 75 & 19,37 & 41122,91 & 548,31 & 22,40 & 57123,96 & 761,65 & 25,51 & 77972,15 & 1039,63 \\
\hline 76 & 19,54 & 41698,96 & 548,67 & 22,58 & 57844,44 & 761,11 & 25,68 & 78865,10 & 1037,70 \\
\hline 77 & 19,71 & 42267,80 & 548,93 & 22,74 & 58554,93 & 760,45 & 25,85 & 79744,70 & 1035,65 \\
\hline 78 & 19,88 & 42829,52 & 549,10 & 22,91 & 59255,61 & 759,69 & 26,01 & 80611,17 & 1033,48 \\
\hline 79 & $\underline{20,04}$ & 43384,20 & $\underline{549,17}$ & 23,07 & 59946,61 & 758,82 & 26,17 & 81464,77 & 1031,20 \\
\hline 80 & 20.20 & 43931,93 & 549,15 & 23,23 & 60628,09 & 757.85 & 26.33 & 82305,73 & 1028.82 \\
\hline
\end{tabular}

R. Árvore, Viçosa-MG, v.29, n.5, p.711-718, 2005 


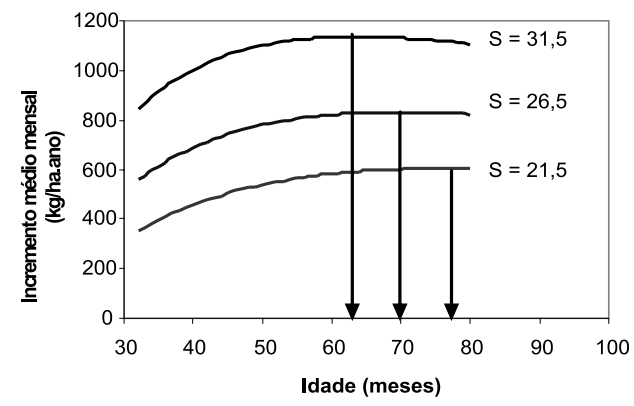

(A)

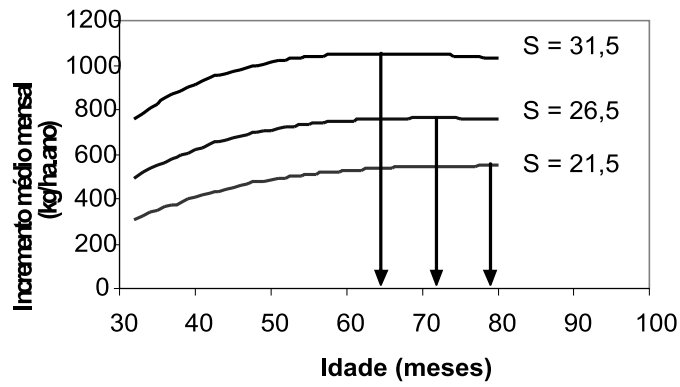

(B)

Figura 4 - Incremento médio mensal do carbono total no fuste (A) e do carbono na madeira (B), nas três classes de índice de local.

Figure 4-Mean monthly increment for total carbon stock outside bark (A) and inside bark (B).

\section{CONCLUSÕES}

Após as análises, obtiveram-se as seguintes conclusões:

- As equações para estimar a quantidade de carbono presente no fuste de árvores individuais de eucalipto, em função do DAP (diâmetro com casca medido a 1,30 $\mathrm{m}$ do solo) e da altura total da árvore (Ht), apresentaramse precisas, com coeficientes de determinação $\left(\mathrm{R}^{2}\right)$ maiores que $97,9 \%$;

- Os modelos de crescimento e produção forneceram estimativas precisas do estoque de carbono, e o modelo de Clutter (1963) apresentou coerência na determinação das idades técnicas de colheita (ITC), em razão da produtividade dos plantios.

\section{REFERÊNCIAS BIBLIOGRÁFICAS}

BROWN, S.; LUGO, A. E. Biomass of tropical forest: A new estimate based on forest volumes. Science, v.223, p.1290-1293, 1984.

BROWN, S.; LUGO, A. E.; CHAPMAN, J. Biomass of tropical tree plantations and its implication for the global carbon budget. Canadian Journal of Forest Research, v.13, p.390-394, 1986.

CAMPOS, J. C. C.; LEITE, H. G. Mensuração Florestal: perguntas e respostas. Viçosa, MG: Universidade Federal de Viçosa, 2002. 407p.

CLUTTER, J.L. Compatible growth and yield models for lobolly pine. Forest Science, v.9, n.3, p.354-371, 1963.

R. Árvore, Viçosa-MG, v.29, n.5, p.711-718, 2005
COOPER, C. F. Carbon storage in managed forest. Canadian Journal of Forest Research, v.13, n.1, p.155-165, 1983.

HASENAUER, H. Princípios para a modelagem de ecossistemas florestais. Revista Ciência \& Ambiente, n. 20, p. 53-69, 2000.

HIGUCHI, N.; CARVALHO JR., J. A. Fitomassa, e conteúdo de carbono de espécies arbóreas da Amazônia. In: SEMINÁRIO EMISSÃO X SEQÜESTRO DE CO2 - uma nova oportunidade de negócios para o Brasil, 1994, Rio de Janeiro.

Anais... Rio de Janeiro: Companhia Vale do Rio Doce, 1994. 125-153p.

HUSCH, B.; MILLER, C. I.; BEERS, T. W. Forest mensuration. 3.ed. Florida: Krieger Publishing Company, 1993. 402 p.

SCHROEDER, P. Carbon storage potential of short rotation tropical tree plantations. Forest Ecology and Management, v.50, p.31-41, 1992.

SCHUMACHER, F.X.; HALL, F. S. Logarithmic expression of timber-tree volume. Journal of Agricultural Research, v.47, n.9, p.719-734, 1933.

SOARES, C. P. B.; OLIVEIRA, M. L. R. Equações para estimar a quantidade de carbono na parte aérea de árvores de eucalipto em Viçosa, Minas Gerais.

Revista Árvore, v.26, n.5, p.533-539, 2002.

VITAL, B. R. Métodos de determinação da densidade da madeira. Boletim Técnico SIF, n. 1, p. 1-21, 1984. 